\title{
A Three-Dimensional Constitutive Modeling for Shape Memory Alloys Considering Two-Way Shape Memory Effect and Transformation-Induced Plasticity
}

\author{
Lei $\mathrm{Xu} *$ \\ Texas A $3 M$ University, College Station, TX 77843, USA \\ Alexandros Solomou ${ }^{\dagger}$ \\ Texas A83M University, College Station, TX 77843, USA \\ Dimitris Lagoudas * \\ Texas A $8 M$ M University, College Station, TX 77843, USA
}

\begin{abstract}
Shape memory alloys (SMAs), since the discovery of their shape memory effect, have been intensively investigated as actuators for the past several decades. Due to their high actuation energy density compared to other active materials, their current and potential applications in the biomedical, aerospace, automobile and energy fields are expanding rapidly. Prior to be used as actuators, SMAs are usually subjected to a training process (i.e. thermal cycling under isobaric conditions) to stabilize their behavior. During the training process, permanent changes are introduced in the microstructure of the material which results in the generation of internal stresses and a large amount of irrecoverable Transformation Induced Plastic strain (TRIP). The generated internal stresses along with a potential thermal loading provide the driving force to induce the oriented phase transformation so that the SMA-based actuators are able to exhibit the Two-Way Shape Memory Effect (TWSME) without applying external bias load. To predict this intrinsic phenomenon, a three-dimensional phenomenological constitutive model for untrained SMAs is presented. The proposed model utilizes the martensitic volume fraction, transformation strain, TRIP strain, and internal stress as internal state variables so that it is able to account for the evolution of TRIP strain and the TWSME for untrained SMAs under cyclic thermomechanical loading conditions. In the end, boundary value problems considering an untrained SMA material under isothermal/isobaric cyclic loading are solved and the predicted cyclic response is compared against available experimental data to demonstrate the proposed capabilities. The proposed model is anticipated to be further validated against additional experimental data for NiTiHf SMAs under general 3-D loading conditions. The validated model will be utilized as an efficient tool for the design and analysis of SMA-based actuators, such as SMA beams and torque tubes, which are intended for the realization of the future supersonic transport aircraft with morphing capabilities to reduce the sonic boom noise.
\end{abstract}

\footnotetext{
*Graduate Research Assistant, Aerospace Engineering, College Station, TX 77843, USA, Student member.

${ }^{\dagger}$ Post-doctoral Research Associate, Aerospace Engineering, College Station, TX 77843, USA

$\ddagger$ Distinguished Professor,Aerospace Engineering,College Station, TX 77843, USA, Faculty member.
} 


\section{Nomenclature}

\begin{tabular}{|c|c|c|c|}
\hline $\mathcal{S}$ & Forth order compliance tensor & $M_{s}$ & Martensite transformation start temp. \\
\hline $\mathcal{S}^{A}$ & Forth order compliance tensor of austenite & $M_{f}$ & Martensite transformation finish temp. \\
\hline $\mathcal{S}^{M}$ & Forth order compliance tensor of martensite & $G$ & Gibbs free energy \\
\hline$\Delta \mathcal{S}$ & Phase difference of compliance tensor & $H^{\text {cur }}$ & Current transformation strain \\
\hline & between austenite and martensite & $H^{\max }$ & Maximum transformation strain \\
\hline$\Lambda$ & Transformation direction tensor & $T$ & Temperature \\
\hline$\Lambda_{f w d}$ & Forward transformation direction tensor & $T_{0}$ & Temperature at reference point \\
\hline$\Lambda_{\text {rev }}$ & Reverse transformation direction tensor & $Y$ & Critical thermodynamic driving force \\
\hline$\Lambda^{p}$ & TRIP strain direction tensor & $Y_{0}$ & Reference thermodynamic driving force \\
\hline$\Lambda_{f w d}^{p}$ & Forward TRIP strain direction tensor & $a_{1}, a_{2}, a_{3}$ & Material parameters in hardening function \\
\hline$\Lambda_{f w d}^{p}$ & Reverse TRIP strain direction tensor & $c$ & Specific heat \\
\hline & Transformation strain & $f(\xi)$ & Smooth hardening function \\
\hline$\varepsilon^{p}$ & TRIP strain & $n_{1}, n_{2}$ & Smooth hardening parameters \\
\hline$\varepsilon^{t-r}$ & Transformation strain at reverse point & $n_{3}, n_{4}$ & Smooth hardening parameters \\
\hline$\Upsilon$ & Set of internal state variables & $k_{t}$ & Material parameter in $H^{\text {cur }}$ curve \\
\hline$\sigma$ & Cauchy stress & $s$ & Effective specific entropy \\
\hline $\boldsymbol{\sigma}_{f}$ & Effective stress & $s_{0}$ & Specific entropy at reference state \\
\hline$\sigma^{\prime}$ & Deviatoric part of Cauchy stress & $\Delta s_{0}$ & Difference of specific entropy \\
\hline $\boldsymbol{\sigma}_{f}^{\prime}$ & Deviatoric part of effective stress & $u$ & Effective internal energy \\
\hline $\bar{\sigma}$ & von Mises equivalent Cauchy stress & $u_{0}$ & Internal energy at reference state \\
\hline $\bar{\sigma}_{f}$ & von Mises equivalent effective stress & $\Delta u_{0}$ & Phase difference of internal energy \\
\hline$\beta$ & Internal stress & $\Phi$ & Transformation function \\
\hline$\alpha$ & Effective thermal expansion tensor & $\sigma_{b}$ & Maximum internal stress magnitude \\
\hline$\Delta \alpha$ & Phase difference of thermal expansion & $\rho$ & Density \\
\hline$A_{s}$ & Austenite transformation start temp. & $\xi$ & Martensitic volume fraction \\
\hline$A_{f}$ & Austenite transformation finish temp. & $\xi^{d}$ & Detwnnied martensitic volume fraction \\
\hline$C_{1}^{p}$ & TRIP strain evolution parameter & $\pi$ & Thermodynamic driving force \\
\hline$C_{2}^{p}$ & TRIP strain evolution parameter & $\lambda_{1}$ & Internal stress evolution parameter \\
\hline & Smooth hardening function parameter & $\zeta^{d}$ & Accumulated detwinned martensitic volum \\
\hline
\end{tabular}

\section{INTRODUCTION}

SMAs are metallic alloys with the ability to recover their pre-defined shapes when subjected to appropriate thermo-mechanical loadings. 1 Since the discovery of their shape memory effect, SMAs have been intensively investigated as actuators to realize actively controlled morphing structures during the past several decades. Due to their high actuation energy density compared to other active materials, such as shape memory polymers and piezoelectrics, the current and potential applications of SMA-based actuators in the biomedical, aerospace, automobile and energy fields are expanding rapidly. For instance, an SMA-based beam component was used as a bending actuator to morph the engine outer shell geometry so that desired aerodynamic conditions can be achieved for the airplane. ${ }^{2}$ Another example is a SMA-based torque tube that was used as a rotation actuator to deploy and contract a solar panel for small satellite ${ }^{3}$ SMA torque tubes are also used as rotational actuator installed on a Boeing airplane to morph the wing flaps at the trailing edge in a full scale flight test $4 \sqrt{5}$

Experimental evidences indicate that the response of SMAs subjected to cyclic thermomechanical loading conditions is non-stable and TRIP strains are accumulated during the process.6 ${ }^{6}$ The reason is repeated phase transformation induces significant distortion located at the austenite-martensite interfaces and grain boundaries, especially during the very first thermomechanical cycles. This distortion drives dislocation activity resulting in an observable macroscopic transformation-induced plastic strain, which occurs at effective stress levels much lower than the plastic yield limit of the material. In general, more than $20 \%$ TRIP strain may accumulate during the lifetime of an SMA component.9 As a result, a training procedure, i.e., thermal cycling under external bias load, is commonly performed on untrained SMAs components to stabilize their response before they are used as actuators. 
A substantial number of consitutive models have been developed to predict the stable response of trained SMAs. A thorough literature review of the available work can be found from literatures 1] 10 16 In the present work, a new three-dimensional SMA phenomenological constitutive model is developed to capture the evolutionary response of untrained SMAs. The proposed model predicts the traditional thermomechanically induced phase transformations, and accounts for the evolutionary material response due to the accumulation of TRIP strains. This model is also able to predict the generation of internal stresses due to the presence of the TRIP strain, which in return leads to its capability to predict the TWSME exhibited by trained SMAs under stress-free thermal cycling loading conditions. The martensitic volume fraction, transformation strain tensor, TRIP strain tensor, and internal stress tensor are used as internal state variables in the proposed modeling framework, and the predictions provided by the model are compared with the corresponding experimental results to demonstrate its capabilities. The proposed model is anticipated to be further validated against additional experimental data for NiTiHf SMAs under general complex loading conditions. The validated model will be utilized as an efficient tool for the design and analysis of SMA-based actuators, such as SMA beams and torque tubes, which are intended to be integrated with the future supersonic transport aircraft that can adapt their shape to reduce the sonic boom noise from takeoff to landing.

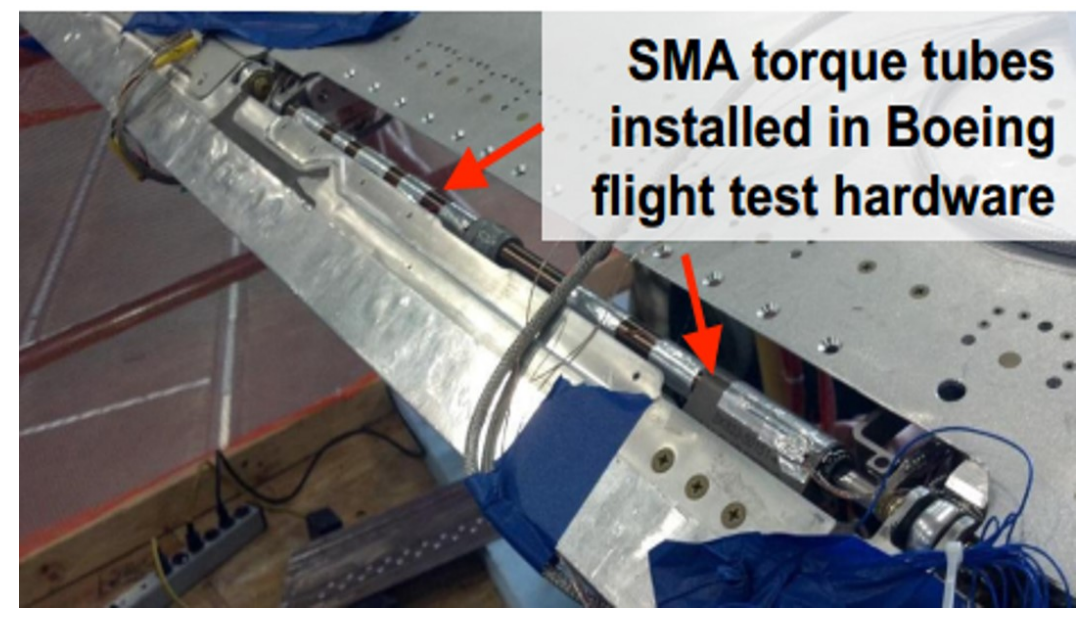

Figure 1: An SMA torque tube actuator installed on a Boeing airplane to rotate the trailing edge wing flaps for a full scale flight test $4 / 5$

\section{Model Formulation}

\section{A. Constitutive model for SMAs}

\section{Thermodynamic potential and constitutive equations}

Based on the work of Lagoudas et al.$^{8}$ and Xu et al., 17 the Gibbs free energy $G$ is proposed to be a continuous function dependent on Cauchy stress tensor $\boldsymbol{\sigma}$, temperature $T$, and a set of internal state variables $\boldsymbol{\Upsilon}=\left\{\boldsymbol{\varepsilon}^{t}, \boldsymbol{\varepsilon}^{p}, \boldsymbol{\beta}, \xi\right\}$, i.e., the transformation strain tensor $\boldsymbol{\varepsilon}^{t}$, the TRIP strain tensor $\boldsymbol{\varepsilon}^{t p}$, the internal stress tensor $\boldsymbol{\beta}$, and the martensitic volume fraction scalar $\xi$, respectively. The $\varepsilon^{t}$ is used to account for the inelastic strain caused by the phase transformation, $\varepsilon^{p}$ is used to represent the large accumulated plastic strain induced by phase transformation, $\boldsymbol{\beta}$ is used for the generated internal stress tensor during training process, and the martensitic volume fraction $\xi$ (ranging $0 \leqslant \xi \leqslant 1$ ) is used for differentiating the two different phases in the SMAs system. The explicit expression for $G$ is given as follows,

$$
\begin{array}{r}
G=-\frac{1}{2 \rho} \boldsymbol{\sigma}: \mathcal{S} \boldsymbol{\sigma}-\frac{1}{\rho} \boldsymbol{\sigma}:\left[\boldsymbol{\alpha}\left(T-T_{0}\right)+\boldsymbol{\varepsilon}^{t}+\boldsymbol{\varepsilon}^{p}\right]-\frac{1}{\rho} \int_{0}^{\xi}\left(\boldsymbol{\beta}: \frac{\partial \varepsilon^{t}}{\partial \xi}\right) d \xi+c\left[\left(T-T_{0}\right)-T \ln \left(\frac{T}{T_{0}}\right)\right]- \\
s_{0} T+u_{0}+f(\xi)
\end{array}
$$


in which, $\mathcal{S}$ is the effective fourth-order compliance tensor calculated by equation $(2), \boldsymbol{\alpha}$ is the effective second order thermal expansion tensor, $c$ is effective specific heat, $s_{0}, u_{0}$ are effective specific entropy and effective specific internal energy at reference state, respectively, $T$ is the current temperature and $T_{0}$ is the reference temperature, $f(\xi)$ is a smooth hardening function.

$$
\mathcal{S}(\xi)=\mathcal{S}^{A}+\xi\left(\mathcal{S}^{M}-\mathcal{S}^{A}\right)=\mathcal{S}^{A}+\xi \Delta \mathcal{S}
$$

On basis of the proposed Gibbs free energy, following classical thermodynamic principles and standard Coleman-Noll procedure, the following constitutive relations can be obtained,

$$
\begin{gathered}
\boldsymbol{\varepsilon}=-\rho \frac{\partial G}{\partial \boldsymbol{\sigma}}=\mathcal{S} \boldsymbol{\sigma}+\boldsymbol{\alpha}\left(T-T_{0}\right)+\boldsymbol{\varepsilon}^{t}+\boldsymbol{\varepsilon}^{p} \\
s=-\rho_{0} \frac{\partial G}{\partial T}=\frac{1}{\rho} \boldsymbol{\sigma}: \boldsymbol{\alpha}+c \ln \left(\frac{T}{T_{0}}\right)-s_{0}
\end{gathered}
$$

\section{Evolution equation for transformation strain}

In this part, the evolution equation of transformation strain $\varepsilon^{t}$ is presented. Following the principle of maximum dissipation, $\frac{18}{18}$ it is proposed that the rate change of the transformation strain $\dot{\varepsilon}^{t}$ is proportional to the rate change of the martenstitic volume fraction $\dot{\xi}$, which results in the following evolution relationship between $\xi$ and $\varepsilon^{t}$,

$$
\dot{\varepsilon}^{t}=\boldsymbol{\Lambda} \dot{\xi}, \quad \boldsymbol{\Lambda}=\left\{\begin{array}{l}
\boldsymbol{\Lambda}_{f w d}, \dot{\xi}>0, \\
\boldsymbol{\Lambda}_{r e v}, \dot{\xi}<0,
\end{array}\right.
$$

where $\boldsymbol{\Lambda}_{f w d}$ is called the forward transformation direction tensor and $\boldsymbol{\Lambda}_{r e v}$ is the reverse transformation direction tensor, they are defined as follows respectively,

$$
\boldsymbol{\Lambda}_{f w d}=\frac{3}{2} H^{c u r} \frac{\boldsymbol{\sigma}_{f}^{\prime}}{\overline{\boldsymbol{\sigma}}_{f}} \boldsymbol{\Lambda}_{r e v}=\frac{\varepsilon^{t-r}}{\xi^{r}} .
$$

in which $\boldsymbol{\sigma}_{f}$ is called the effective stress tensor defined in equation $(7), \varepsilon^{t-r}$ and $\xi^{r}$ denotes the value of transformation strain and martensitic volume fraction at the reverse transformation starting point. The internal stress tensor $\boldsymbol{\beta}$ generated during cyclic loading is introduced in section 4.

$$
\sigma_{f}=\boldsymbol{\sigma}+\boldsymbol{\beta}
$$

$H^{\text {cur }}$ denotes the current maximum transformation strain at current stress state. Based on the observation from experimental results, the transformation strain is usually not a constant value but exponentially depends on current material stress state. The reason is that the austenitic phase can be transformed into oriented/twinned martensitic phase depending on the current stress state. Therefore an exponential function $H^{\text {cur }}$ based on current stress state is introduced to obtain the current transformation strain magnitude as shown in equation (8), where $H^{\max }$ is the maximum (or saturated) transformation strain and $k_{t}$ is a material curve fitting parameter.

$$
H^{\text {cur }}(\boldsymbol{\sigma})=H^{\max }\left(1-e^{-k_{t} \overline{\boldsymbol{\sigma}}_{f}}\right)
$$

$\boldsymbol{\sigma}_{f}^{\prime}$ is the deviatoric part of the effective stress tensor, i.e., $\boldsymbol{\sigma}_{f}^{\prime}=\boldsymbol{\sigma}_{f}-\frac{1}{3} \operatorname{tr}\left(\boldsymbol{\sigma}_{f}\right) * \mathbf{1}$, in which $\mathbf{1}$ is the second order identity tensor. The von mises equivalent effective stress $\bar{\sigma}_{f}$ is given as,

$$
\bar{\sigma}_{f}=\sqrt{\frac{3}{2} \sigma_{f}^{\prime}: \boldsymbol{\sigma}_{f}^{\prime}}
$$

\section{Evolution equation for TRIP strain}

In this part, the evolution equation for the TRIP strain $\varepsilon^{p}$ is presented. Based on the observation from experimental results, the TRIP strain is evolved with respect to the loading cycles. Following the evolution 
equation proposed by Lagoudas and Entchev ${ }^{8}$ it is proposed that the rate change of the TRIP strain $\dot{\varepsilon}^{p}$ is proportional to the rate change of the martenstitic volume fraction $\dot{\xi}$, which is expressed as follows,

$$
\dot{\varepsilon}^{p}=\Lambda^{p} \dot{\xi}, \quad \boldsymbol{\Lambda}^{p}=\left\{\begin{array}{l}
\boldsymbol{\Lambda}_{f w d}^{p}, \dot{\xi}>0, \\
\boldsymbol{\Lambda}_{r e v}^{p}, \dot{\xi}<0,
\end{array}\right.
$$

in which $\boldsymbol{\Lambda}_{f w d}^{p}$ is called the forward TRIP direction tensor, $\boldsymbol{\Lambda}_{r e v}^{p}$ is the reverse TRIP direction tensor. Their explicit expressions are defined as follows,

$$
\boldsymbol{\Lambda}_{f w d}^{p}=\frac{3}{2} C_{1}^{p} \frac{H^{c u r}(\boldsymbol{\sigma})}{H^{\max }} \frac{\boldsymbol{\sigma}_{f}^{\prime}}{\overline{\boldsymbol{\sigma}}_{f}} e^{-\frac{\zeta^{d}}{C_{2}^{p}}} ; \quad \boldsymbol{\Lambda}_{\text {rev }}^{p}=-C_{1}^{p} \frac{H^{c u r}(\boldsymbol{\sigma})}{H^{\max }} \frac{\varepsilon^{t-r}}{\xi^{r}} e^{-\frac{\zeta^{d}}{C_{2}^{p}}} .
$$

The evolution of TRIP strain assumes that only the orientated (or called detwinned) martensitic phase transformation contributes to the generation of TRIP stain while the twinned martensitic phase transformation does not ${ }^{8}$ This assumption is reflected in the above TRIP direction tensor by the ratio of $H^{\text {cur }}$ over $H^{\max }$ and a term $\zeta^{d}$ called the accumulated detwinned martensitic volume fraction in the exponential function. The explicit expression of $\zeta^{d}$ is defined as follows,

$$
\zeta^{d}=\int_{0}^{t}\left|\dot{\xi}^{d}(t)\right| d t
$$

in which the detwinned martensitic volume fraction $\xi^{d}$ is obtained by,

$$
\xi^{d}=\frac{H^{c u r}(\boldsymbol{\sigma})}{H^{\max }} \xi
$$

\section{Evolution equation for internal Stress}

As described in section I, the mechanism for promoting TWSME in SMAs is the generation of internal stresses by inducing the microstructure changes (such as dislocation movement, retained martensitic variants, and oriented growth of coherent precipitates) through cyclic loading ${ }^{19}$ These generated oriented internal stress fields along with a potential thermal stimulus are then able to drive the oriented martensitic phase transformation when SMAs subject to stress free conditions resulting in the so-called TWSME. In oder to model this process, a second order internal stress tensor $\boldsymbol{\beta}$ is introduced in this model. The evolution equation of this internal stress is proposed as follows,

$$
\boldsymbol{\beta}=\sigma_{b} \frac{\boldsymbol{\sigma}_{f}}{\overline{\boldsymbol{\sigma}}_{f}}\left(1-e^{-\lambda_{1} \zeta^{d}}\right)
$$

this evolution equation assumes that the internal stress is generated along the same direction of the applied external forces, in which $\sigma_{b}$ is a material parameter representing the maximum (or saturated) internal stress magnitude that can be generated during the training process. $\zeta^{d}$ is the accumulated detwinned martensitic volume fraction described above and $\lambda_{1}$ is a curve fitting parameter indicating how the internal stress evolves with respect to the number of loading cycle.

\section{Smooth hardening function}

A smooth hardening function $f(\xi)$ is proposed in equation 15 to account for the hardening effects associated with the transformation process, $\frac{1]}{1}$ where three additional intermediate material parameters $a_{1}, a_{2}, a_{3}$ and four curve fitting parameters $n_{1}, n_{2}, n_{3}, n_{4}$ are introduced to better treat the smooth transition behaviors during the initiation and completion of transformations.

$$
f(\xi)=\left\{\begin{array}{l}
\frac{1}{2} a_{1}\left(\xi+\frac{\xi^{n_{1}+1}}{n_{1}+1}+\frac{(1-\xi)^{n_{2}+1}}{n_{2}+1}\right)+a_{3} \xi, \dot{\xi}>0, \\
\frac{1}{2} a_{2}\left(\xi+\frac{\xi^{n_{3}+1}}{n_{3}+1}+\frac{(1-\xi)^{n_{4}+1}}{n_{4}+1}\right)-a_{3} \xi, \dot{\xi}<0
\end{array}\right.
$$




\section{Transformation function}

The objective of this section is to define a proper transformation criterion to determine the occurrence of the phase transformations. After the introduction of evolution equation for internal state variables, on basis of the $2^{\text {nd }}$ thermodynamic principle, substituting the evolution equation 5 and 10 into the reduced form dissipation inequality resulting in the following expression,

$$
\left(\boldsymbol{\sigma}: \boldsymbol{\Lambda}+\boldsymbol{\beta}: \boldsymbol{\Lambda}+\boldsymbol{\sigma}: \boldsymbol{\Lambda}^{p}-\rho \frac{\partial G}{\partial \xi}\right) \dot{\xi}=\pi \dot{\xi} \geqslant 0
$$

where the quantity $\pi$ is called the general thermodynamic driving force conjugated to $\xi$. Substituting the equation (1) of Gibbs free energy into equation (16), the explicit expression for the thermodynamic driving force $\pi$ is obtained as,

$$
\begin{aligned}
& \pi=(\boldsymbol{\sigma}+\boldsymbol{\beta}): \boldsymbol{\Lambda}+\boldsymbol{\sigma}: \boldsymbol{\Lambda}^{p}+\frac{1}{2} \boldsymbol{\sigma}: \Delta \mathcal{S} \boldsymbol{\sigma}+\boldsymbol{\sigma}: \Delta \boldsymbol{\alpha}\left(T-T_{0}\right)+\rho \Delta s_{0} T-\rho \Delta c\left[T-T_{0}-T \ln \left(\frac{T}{T_{0}}\right)\right]- \\
& \rho \Delta u_{0}-\frac{\partial f}{\partial \xi}
\end{aligned}
$$

where $\Delta \mathcal{S}, \Delta \boldsymbol{\alpha}, \Delta c, \Delta s_{0}$, and $\Delta u_{0}$ are the phase differences on compliance tensor, thermal expansion tensor, specific heat, reference entropy and reference internal energy. It can be observed that the thermodynamic driving force $\pi$ is a function of stress $\boldsymbol{\sigma}$, temperature $T$ and martenstic volume fraction $\xi$. This indicates that the phase transformation process can be activated by two independent sources, namely either the stress or temperature, which correlates quite well with the experimentally observed stress-induced and thermallyinduced phase transformations in SMAs. To proceed to the goal of defining a transfomration criteria, it is assumed that whenever the thermodynamic driving force $\pi$ reaches a critical value $Y(-Y)$, the forward (reverse) phase transformation takes place. Therefore a transformation function $\Phi$ can be defined as the transformation criteria to determine the transformation occurrence as follows,

$$
\Phi=\left\{\begin{array}{c}
\pi-Y, \dot{\xi}>0, \\
-\pi-Y, \dot{\xi}<0,
\end{array}\right.
$$

In the continuous development of the established SMA model, 11 a reference critical value $Y_{0}$ and an additional parameter $\mathrm{D}$ were introduced into $Y$ such that the model can capture the different slopes $C_{A}, C_{M}$ in the effective stress-temperature phase diagram. This development is also adopted here.

$$
Y(\boldsymbol{\sigma})=\left\{\begin{array}{l}
Y_{0}+D \boldsymbol{\sigma}: \boldsymbol{\Lambda}_{f w d}, \dot{\xi}>0, \\
Y_{0}+D \boldsymbol{\sigma}: \boldsymbol{\Lambda}_{r e v}, \dot{\xi}<0,
\end{array}\right.
$$

As a consequence of the maximum dissipation principle, the so-called Kuhn-Tucker constraints are applied on the proposed model as follows, 18

$$
\begin{aligned}
& \dot{\xi} \geqslant 0 ; \quad \Phi(\boldsymbol{\sigma}, T, \xi)=\pi-Y \leqslant 0 ; \quad \Phi \dot{\xi}=0 ; \quad(\mathbf{A} \Rightarrow \mathbf{M}) \\
& \dot{\xi} \leqslant 0 ; \quad \Phi(\boldsymbol{\sigma}, T, \xi)=-\pi-Y \leqslant 0 ; \quad \Phi \dot{\xi}=0 ; \quad(\mathbf{M} \Rightarrow \mathbf{A})
\end{aligned}
$$

\section{Determination of material parameters}

There are three groups of material parameters need be calibrated described as table 1. Elastic modulus $E_{A}, E_{M}$ can be determined through a pseudoelastic test by calculating the slopes at initial loading and unloading stage. A widely accepted assumption for Poisson's ratio is $\nu_{A}=\nu_{M}$ with reported value of 0.33 found in literature ${ }^{6}$ It is also reasonable to assume that the thermal expansion coefficient for two phases are equal $\alpha_{A}=\alpha_{M}$ which can be calibrated through an isobaric actuation experiment. Other material constants characterizing stable transformation cycles $\left(C_{A}, C_{M}, M_{s}, M_{f}, A_{s}, A_{f}\right)$ can be calibrated through the stress-temperature phase diagram. Next, material parameters related to transformation strain and smooth

hardening effects are discussed. The maximum transformation strain $H^{\max }$ can be directly determined from experimental data and the value of parameter $k_{t}$ are chosen to best fit the $H^{c u r}$ curve. Coefficients 
$n_{1}, n_{2}, n_{3}, n_{4}$ without specific physical material meanings are determined to best match the smoothness corners of transformation hysteresis. Material parameters related to TRIP strain and internal stress are determined from experiment data. There are also seven additional parameters $\left(\rho \Delta s_{0}, \rho \Delta u_{0}, a_{1}, a_{2}, a_{3}, Y_{0}\right.$ and $\left.D\right)$ that can be written by the aforementioned material parameters. Referring to the literature, $\frac{11}{1}$ the first 5 intermediate material parameters $\left(a_{1}, a_{2}, a_{3}, \rho \Delta u_{0}, Y_{0}\right)$ can be expressed in terms of the previous directly measured material parameters as,

$$
\left\{\begin{array}{l}
a_{1}=\rho \Delta s_{0}\left(M_{f}-M_{s}\right) ; \quad a_{2}=\rho \Delta s_{0}\left(A_{s}-A_{f}\right) \\
a_{3}=\frac{1}{4} a_{2}\left(1+\frac{1}{n_{3}+1}\right)-\frac{1}{4} a_{1}\left(1+\frac{1}{n_{1}+1}\right) \\
\rho \Delta u_{0}=\frac{1}{2} \rho \Delta s_{0}\left(M_{s}+A_{f}\right)+\beta \Lambda \\
Y_{0}=\frac{1}{2} \rho \Delta s_{0}\left(M_{s}-A_{f}\right)-a_{3}
\end{array}\right.
$$

the additional two intermediate material parameters $\rho \Delta s_{0}$ and $D$ can be expressed as,

$$
\left\{\begin{array}{l}
D=\frac{\left(C_{M}-C_{A}\right)\left[H^{c u r}+\sigma \partial_{\sigma} H^{c u r}+\beta \partial_{\sigma} H^{c u r}+\sigma\left(\frac{1}{E_{M}}-\frac{1}{E_{A}}\right)\right]}{\left(C_{M}+C_{A}\right)\left(H^{c u r}+\sigma \partial_{\sigma} H^{c u r}\right)}+\frac{C_{1}^{p}}{H^{\text {max }}} e^{-\frac{\zeta^{d}}{C_{2}^{p}}} \\
\rho \Delta s_{0}=-\frac{-2 C_{M} C_{A}\left[H^{c u r}+\sigma \partial_{\sigma} H^{c u r}+\beta \partial_{\sigma} H^{c u r}+\sigma\left(\frac{1}{E_{M}}-\frac{1}{E_{A}}\right)\right]}{C_{M}+C_{A}}
\end{array}\right.
$$

\section{NUMERICAL RESULTS}

The model has been proposed in the previous sections and implemented though an user-defined material subroutine(UMAT) under the commercial finite element software Abaqus. To demonstrate the capabilities of the proposed model to capture the TRIP strains and internal stresses generated during thermomechanical cyclic loading, two boundary value problems (BVPs) are investigated here and the model predictions are compared against the available experimental data. The first problem analyzes the response of a NiTi SMA under isothermal loading conditions subjected to 50 mechanical cycles. In this BVP, the stress-induced phase transformation is simulated and the pseudoelastic stress-strain curves are obtained. The second BVP analyzes the respone of a NiTi SMA under isobaric loading condition subjected to 100 thermal cycles. The thermally-induced phase transformation is simulated and the actuation strain-temperature curves are predicted. The simulations are performed by using the commercial finite element software Abaqus 6.14 .20

\section{A. Isothermal Loading Case}

\section{SMAs under tensile loading}

The first BVP considers an untrained NiTI SMA loaded under isothermal loading conditions and subjected to 50 mechanical cycles. During the mechanical loading cycles the material is loaded up to $550 \mathrm{MPa}$ and then is unloaded to $0 \mathrm{MPa}$. The temperature kept at a constant at $360 \mathrm{~K}$ throughout the entire process. During this process, the material undergoes a forward stress-induced phase transformation during the mechanical loading phase followed by a reverse phase transformation during the unloading phase. The material parameters used in this simulation are summarized in table 1.

The stress-strain curves obtained by the propsed model are compared with the available experimental data ${ }^{1}$ As shown in figure 2 the NiTi SMA accumulated a large amount of irrecoverable TRIP strain from the $1^{\text {st }}$ cycle to the $50^{\text {th }}$ cycle and this behavior is well captured by the proposed model. Moreover, it is interesting to note that the model was able to capture the experimentally observed decrease of the stress level at which the forward phase transformation starts as a result of the mechanical cycling. This intrinsic behavior predicted by the proposed model using the internal stress term in the formulation of the model.The TRIP strain accumulated with respect to number of loading cycles is also plotted in figure 3 It is shown that the TRIP strain generated drastically within the initial 30 loading cycles but has stabilized afterwards. 
Table 1: Material parameters used for the NiTi SMA subjected to isothermal loading conditions ${ }^{1}$

\begin{tabular}{c|lr|lr}
\hline Type & Parameter & Value & Parameter & Value \\
\hline & $E_{A}$ & $70[\mathrm{GPa}]$ & $C_{A}$ & $3.4[\mathrm{MPa} / \mathrm{K}]$ \\
Major material parameters & $E_{M}$ & $50[\mathrm{GPa}]$ & $C_{M}$ & $3.4[\mathrm{MPa} / \mathrm{K}]$ \\
10 & $\nu_{A}=\nu_{M}$ & 0.33 & $M_{s}$ & $264[\mathrm{~K}]$ \\
& $\alpha_{A}=\alpha_{M}$ & $2.2 \times 10^{-5}\left[\mathrm{~K}^{-1}\right]$ & $M_{f}$ & $160[\mathrm{~K}]$ \\
& $H^{\text {max }}$ & 0.05 & $A_{s}$ & $217[\mathrm{~K}]$ \\
& $k_{t}$ & 0.00752 & $A_{f}$ & $290[\mathrm{~K}]$ \\
\hline Smooth hardening parameters & $n_{1}$ & 0.2 & $n_{3}$ & 0.4 \\
6 & $n_{2}$ & 0.3 & $n_{4}$ & 0.5 \\
\hline \multirow{3}{*}{ TRIP and Internal stress parameters } & $\sigma_{b}$ & $100[\mathrm{MPa}]$ & $\lambda_{1}$ & 3.5 \\
4 & $C_{1}^{p}$ & $3.6 \times 10^{-3}$ & & \\
\hline
\end{tabular}

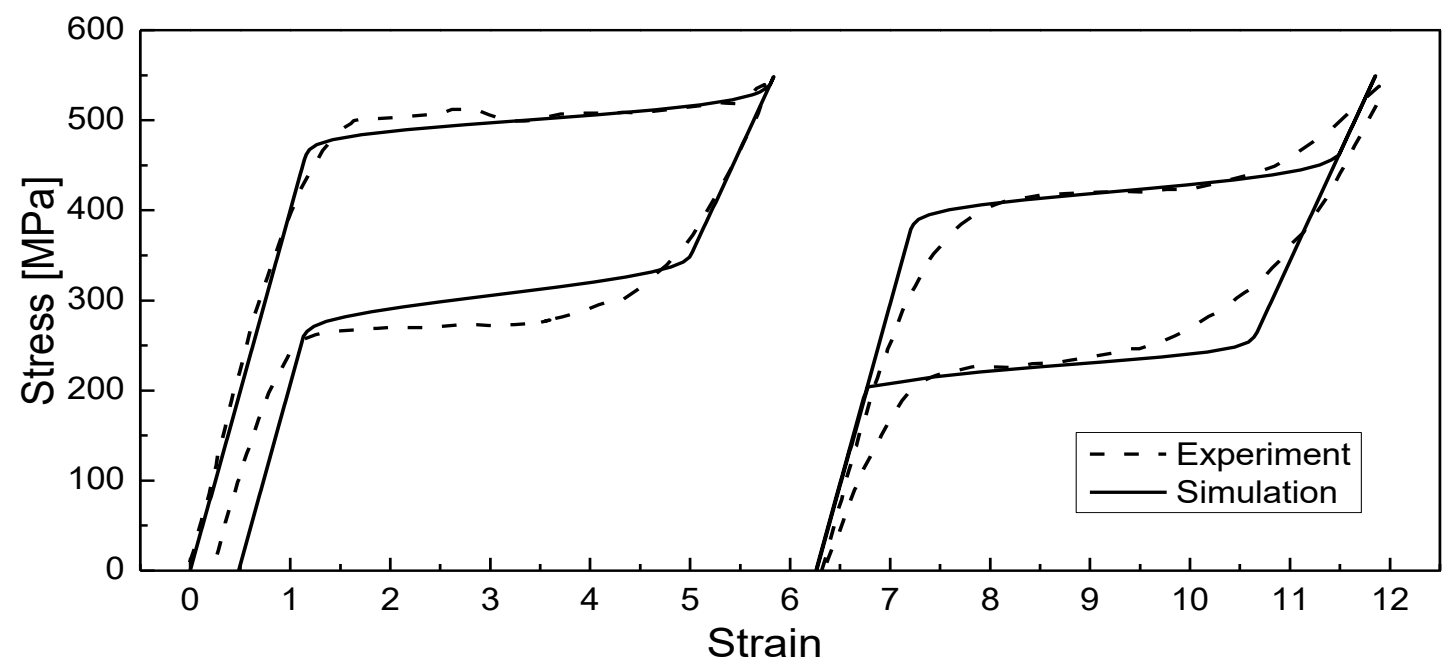

Figure 2: The $1^{\text {st }}$ and $50^{\text {th }}$ pseudoelastic stress-strain response of the untrained NiTi under $550 \mathrm{MPa}$ uniaxial tensile loading.

The TRIP strain in the $1^{\text {st }}$ cycle is around $0.7 \%$ and with a saturated value around $6.5 \%$ after 30 cycles. The predicted TRIP evolution result through the proposed model is in good agreement with the experimental data.

\section{B. Isobaric Loading Case}

The second BVP considers an untrained SMA loaded under isobaric loading conditions and subjected to 100 thermal cycles. To this end, the SMA is initially loaded under a constant tensile load of $200 \mathrm{MPa}$ and then undergoes thermal cycles where the temperature varies from $30^{\circ} \mathrm{C}$ to $165^{\circ} \mathrm{C}$ for 100 cycles. During the aforementioned loading procedure the SMA undergoes a forward thermally-induced phase transformation followed by a reverse phase transformation. Once the 100 thermal cycles are completed, 10 additional thermal cycles under stress-free conditions are performed to examine the TWSME of the material. The material parameters used in this simulation are summarized in table 2 while further details about the experimental data used are described in Atli et al $\frac{19}{19}$

The experimental result is shown in figure 4 , in which the red curve represents the response of the SMA under bias load while the blue curve indicates its response under stress-free condition. It can be seen that a large amount of TRIP stain (around 10\%) is accumulated during the 100 thermal loading cycles under

$$
8 \text { of } 12
$$




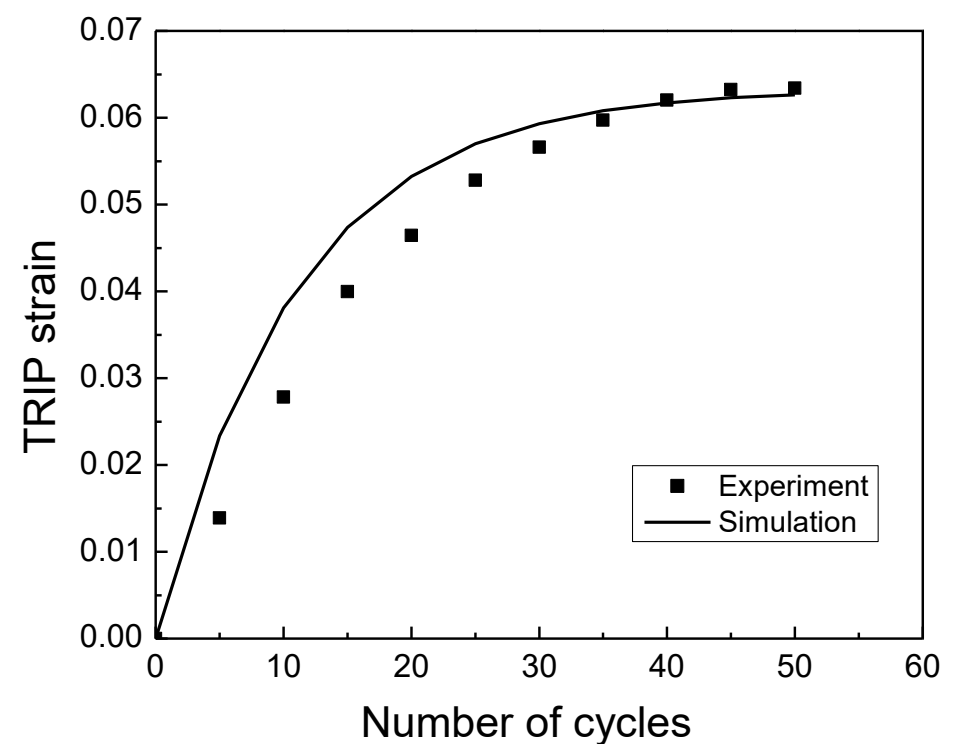

Figure 3: The evolution of TRIP strain for the untrained NiTi material subject to 50 isothermal loading cycles.

Table 2: Material parameters used for the untrained NiTi SMA subject to isobaric loading calibrated based on the experimental data from Atli et al 19

\begin{tabular}{c|lr|lr}
\hline Type & Parameter & Value & Parameter & Value \\
\hline & $E_{A}$ & $22[\mathrm{GPa}]$ & $C_{A}$ & $16[\mathrm{MPa} / \mathrm{K}]$ \\
& $E_{M}$ & $22[\mathrm{GPa}]$ & $C_{M}$ & $8[\mathrm{MPa} / \mathrm{K}]$ \\
Major material parameters & $\nu_{A}=\nu_{M}$ & 0.33 & $M_{s}$ & $340[\mathrm{~K}]$ \\
10 & $\alpha_{A}=\alpha_{M}$ & $2.2 \times 10^{-5}\left[\mathrm{~K}^{-1}\right]$ & $M_{f}$ & $310[\mathrm{~K}]$ \\
& $H^{m a x}$ & 0.045 & $A_{s}$ & $361[\mathrm{~K}]$ \\
& $k_{t}$ & 0.0158 & $A_{f}$ & $395[\mathrm{~K}]$ \\
\hline Smooth hardening parameters & $n_{1}$ & 0.5 & $n_{3}$ & 0.5 \\
6 & $n_{2}$ & $\sigma_{b}$ & $50[\mathrm{MPa}]$ & $\lambda_{1}$ \\
\hline
\end{tabular}

bias load. Furthermore a TWSME is observed in the response of the material under the stress-free thermal loading cycles as depicted by the blue curve. The strain-temperature response for this material, predicted by the proposed model, is shown in figure 5. The predictions are in good agreement with the experimental results. The model successfully captured the evolution on the materials response by predicting adequately the evolution of TRIP strain while it also captured the TWSME of the material using the internal stress term. It is demonstrated that the proposed model can be used as an efficient tool to predict the evolution of the materials response of untrained SMAs and therefore to enable the design and analysis of SMA-based actuators for real engineering applications. 


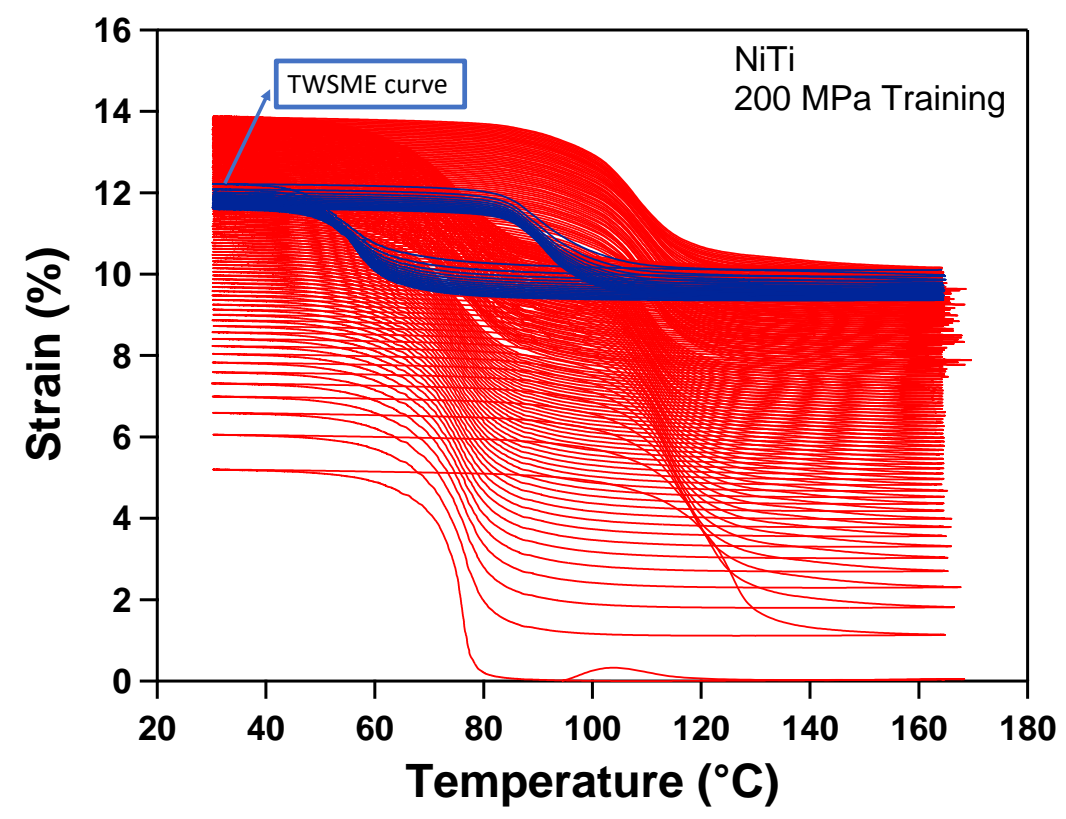

Figure 4: Experimental result shows the TWSME at stress-free conditions for untrained NiTi material after 100 cycles of isobaric loading. The red curve is the response under bias load, and the blude curve indicates the reponse under stress-free condition.

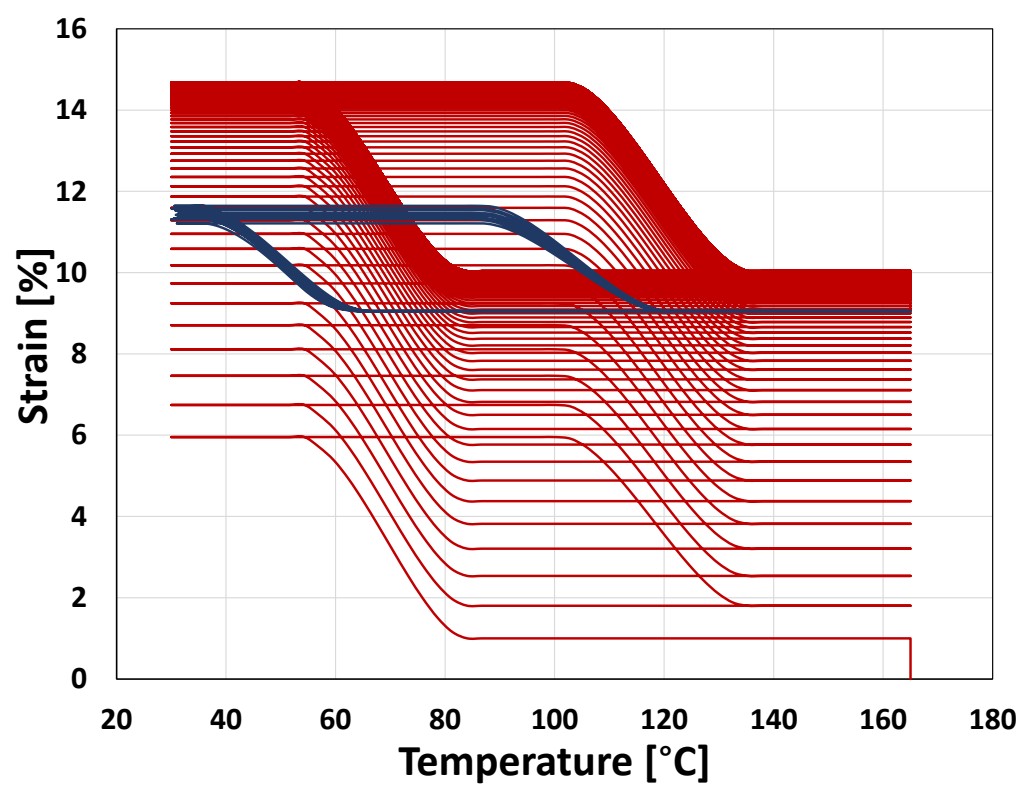

Figure 5: The cyclic temperature-strain response predicted by the proposed model for the untrained NiTi material subject to 100 isobaric loading cycles followed by 10 stress free loading cycles. The red curve is the response under bias load, and the blude curve indicates the reponse under stress-free condition. 


\section{CONCLUSIONS}

This work presents a three-dimensional constitutive model for the martensitic phase transformation of untrained SMAs subject to thermomechanical cyclic loading conditions. The model is able to predict the traditional thermomechanically induced phase transformations in SMAs. Moreover, by introducing a set of additional internal state variables, i.e., TRIP strain tensor and internal stress tensor, the model is further demonstrated to account for the evolution of irrecoverable TRIP strains and the TWSME for untrained SMAs after cyclic loading. The capabilities of the proposed model are demonstrated by comparing its predictions with experimental results. In particular, boundary value problems considering untrained NiTi SMAs subjected to isothermal and isobaric cyclic loading conditions were investigated. It was shown in the isothermal case that the proposed model was able to predict the generation and saturation of TRIP strains, and also to predict the decreasing stress levels required to start the phase transformation. As for the isobaric loading case, it was demonstrated that the proposed model was able to capture the evolution of materials response during the cyclic loading and to reproduce the TWSME for trained SMAs at stress-free condition.

The proposed model is anticipated to be further validated against additional experimental data of NiTi and NiTiHf SMAs under uniaxial and other non-uniform loading conditions. The ultimate objective is to validate the capability of the proposed model to predict the response of SMA-based actuators, such as SMA beams and torque tubes, which are intended to be integrated with the future supersonic transport aircrafts to realize the morphing capabilities to reduce the sonic boom noise.

\section{Acknowledgments}

This work is supported by the National Aeronautics and Space Administration (NASA) through the University Leadership Initiative (ULI) project under the grant number: NNX17AJ96A. The conclusions in this work are solely made by the authors and do not necessarily represent the perspectives of NASA.

\section{References} 2008.

${ }^{1}$ Lagoudas, D. C., Shape memory alloys: modeling and engineering applications, Springer Science \& Business Media,

${ }^{2}$ Hartl, D. J. and Lagoudas, D. C., "Aerospace applications of shape memory alloys," Proceedings of the Institution of Mechanical Engineers, Part G: Journal of Aerospace Engineering, Vol. 221, No. 4, 2007, pp. 535-552.

${ }^{3}$ Wheeler, R., Saunders, R., Pickett, K., Eckert, C., Stroud, H., Fink, T., Gakhar, K., Boyd, J., and Lagoudas, D., "Design of a reconfigurable sma-based solar array deployment mechanism," ASME 2015 Conference on Smart Materials, Adaptive Structures and Intelligent Systems, American Society of Mechanical Engineers, 2015, pp. V001T02A010-V001T02A010.

${ }^{4}$ Mabe, J., Brown, J., and Calkins, F., "Flight test of a shape memory alloy actuated adaptive trailing edge flap, Part 1," Proceedings of SMST 2014 the International Conference on Shape Memory and Superelastic Technologies, 2014.

${ }^{5}$ Calkins, F. and Mabe, J., "Flight Test of a Shape Memory Alloy Actuated Adaptive Trailing Edge Flap," ASME 2016 Conference on Smart Materials, Adaptive Structures and Intelligent Systems, American Society of Mechanical Engineers, 2016, pp. V001T04A007-V001T04A007.

${ }^{6}$ Lagoudas, D. C. and Bo, Z., "Thermomechanical modeling of polycrystalline SMAs under cyclic loading, Part II: Material characterization and experimental results for a stable transformation cycle," International Journal of Engineering Science, Vol. 37, No. 9, 1999, pp. 1141-1173.

${ }^{7}$ Bo, Z. and Lagoudas, D. C., "Thermomechanical modeling of polycrystalline SMAs under cyclic loading, Part III: Evolution of plastic strains and two-way shape memory effect," International Journal of Engineering Science, Vol. 37, No. 9, 1999, pp. 1175-1203.

${ }^{8}$ Lagoudas, D. C. and Entchev, P. B., "Modeling of transformation-induced plasticity and its effect on the behavior of porous shape memory alloys. Part I: constitutive model for fully dense SMAs," Mechanics of Materials, Vol. 36, No. 9, 2004, pp. 865-892.

${ }^{9}$ Wheeler, R. W., Hartl, D. J., Chemisky, Y., and Lagoudas, D. C., "Characterization and Modeling of Thermo-Mechanical Fatigue in Equiatomic NiTi Actuators," ASME 2014 Conference on Smart Materials, Adaptive Structures and Intelligent Systems, American Society of Mechanical Engineers, 2014, pp. V002T02A009-V002T02A009.

${ }^{10}$ Boyd, J. G. and Lagoudas, D. C., "A thermodynamical constitutive model for shape memory materials. Part I. The monolithic shape memory alloy," International Journal of Plasticity, Vol. 12, No. 6, 1996, pp. 805-842.

${ }^{11}$ Lagoudas, D., Hartl, D., Chemisky, Y., Machado, L., and Popov, P., "Constitutive model for the numerical analysis of phase transformation in polycrystalline shape memory alloys," International Journal of Plasticity, Vol. 32, 2012, pp. 155-183.

${ }^{12} \mathrm{Xu}$, L., Baxevanis, T., and Lagoudas, D. C., "A Finite Strain Constitutive Model for Martensitic Transformation in Shape Memory Alloys Based on Logarithmic Strain," 25th AIAA/AHS Adaptive Structures Conference, 2017, p. 0731.

${ }^{13}$ Arghavani, J., Auricchio, F., and Naghdabadi, R., "A finite strain kinematic hardening constitutive model based on Hencky strain: general framework, solution algorithm and application to shape memory alloys," International Journal of Plasticity, Vol. 27, No. 6, 2011, pp. 940-961. 
${ }^{14}$ Thamburaja, P. and Anand, L., "Polycrystalline shape-memory materials: effect of crystallographic texture," Journal of the Mechanics and Physics of Solids, Vol. 49, No. 4, 2001, pp. 709-737.

${ }^{15}$ Patoor, E., Eberhardt, A., and Berveiller, M., "Micromechanical modelling of superelasticity in shape memory alloys," Le Journal de Physique IV, Vol. 6, No. C1, 1996, pp. C1-277.

${ }^{16}$ Patoor, E., Lagoudas, D. C., Entchev, P. B., Brinson, L. C., and Gao, X., "Shape memory alloys, Part I: General properties and modeling of single crystals," Mechanics of materials, Vol. 38, No. 5, 2006, pp. 391-429.

${ }^{17} \mathrm{Xu}$, L., Baxevanis, T., and Lagoudas, D., "A Finite Strain Constitutive Model Considering Transformation Induced Plasticity for Shape Memory Alloys under Cyclic Loading," 8th ECCOMAS Thematic Conference on Smart Structures and Materials, 2017, pp. 1645-1477.

${ }^{18}$ Qidwai, M. and Lagoudas, D., "On thermomechanics and transformation surfaces of polycrystalline NiTi shape memory alloy material," International Journal of Plasticity, Vol. 16, No. 10, 2000, pp. 1309-1343.

${ }^{19}$ Atli, K., Karaman, I., Noebe, R., Bigelow, G., and Gaydosh, D., "Work production using the two-way shape memory effect in NiTi and a Ni-rich NiTiHf high-temperature shape memory alloy," Smart Materials and Structures, Vol. 24, No. 12, 2015, pp. 125023.

${ }^{20}$ Abaqus, V., "6.14 Documentation," Dassault Systemes Simulia Corporation, Vol. 651, 2014. 